\title{
Effect of Wasabi Extract on Inflammatory Response of BV2 Cells Induced by Lipopolysaccharide
}

\author{
Chen Chen ${ }^{1}$, Qiu Yuanyuan ${ }^{2 *}$ \\ ${ }^{1,2}$ School of Electronic Information in Jiangsu, Nantong University, Nantong 226019, China
}

\begin{abstract}
Objective: This study was to investigate the effect of wasabi extract 6-(methylsulfinyl)hexyl isothiocyanate (6-MITC) on preventing neuroinflammation. Methods: The activation of BV2 microglia cell was introduced by LPS to establish an in vitro neuroinflammatory cell model, and the influence of 6-MITC on the survival rate of BV2 cells was deceted by MTT assay and the mRNA expression level of inflammatory factors in each group were deceted by RT-qPCR. Results: 6-MITC can significantly down-regulate mRNA expression levels of the inflammatory factor interleukin-1 $\beta$, IL-6, tumor necrosis factor- $\alpha(\mathrm{TNF}-\alpha)$ and nitric oxide synthase (iNOS) after activation of BV2 cells induced by LPS. Conclusion: 6-MITC can inhibit LPS-induced microglia activation and decrease inflammatory response of BV2 cells.
\end{abstract}

\section{Introduction}

In recent years, both domestic and foreign scholars have concentrated on the metabolites in wasabi tissue and found that wasabi is an edible health care plant rich in biologically active ingredients such as isothiocyanates (ITCs), nitriles, thiocyanates (TCs), epithionitriles and oxazolidinones. Especially, there are many research conducted for isothiocyanates. It has been found that these substances have many healthcare functions such as anti- cancer, anti-thrombosis, anti-oxidation, anti-virus, immune enhancement, and auxiliary blood sugar lowering.4-(methylsulfinyl)butyl isothiocyanate, commonly known as sulforaphane, SFN, has been shown to have neuro- protective effects in a variety of experimental paradigms. As shown in the Fig.1, 6-(methylsulfinyl)hexyl isothio- cyanate (6-MITC) has two more carbon chains than SFN and might be of a stronger biological effect.

Identification of biological pathway experiments by IPA (Ingenuity Pathway Analysis) technique according to Trio et $\mathrm{al}^{1}$ show that the difference in expression gene between 6-MITC and SFN is twice or more. Therefore, 6-MITC is considered to have a greater effect on the regulation of gene expression of IMR-32 cells than SFN. More studies have shown that the structure-activity relationship of wasabi ITCs depends on the methyl chain length of Wasabi ITCs. The longer the methyl chain length of Wasabi ITCs, the stronger the inhibition of Cyclooxy-genase-2 (COX-2) expression is ${ }^{2}$.
A<smiles>CS(=O)CCCCN=C=S</smiles>

B<smiles>CS(=O)CCCCCCCN=C=S</smiles>

6-(methylsulfinyl) hexyl isothiocyanate (6-MITC)

Fig.1 Chemical structures of two ITCs: (A) SFN, (B) 6-MITC

Microglia are the main immune cells widely distrubuted in the central nervous system, and they play an important role in maintenance the central nervous system homeostasis and damage repair. In addition, microglia- mediated neuroinflammatory response holds a key process in the development of cerebral ischemia and neuro- degenerative diseases ${ }^{3}$.

Lipopolysaccharide (LPS) is a major component of the cell wall of Gram-negative bacteria. It is a potent inflammatory response inducer. It is a typical microglia activator that induces microglia phenotype changes and releases inflammation Factor. BV2 cells are mouse glial cell immortalized cell lines, retaining various morphological, characterization and functional characteristics of microglia. In this experiment, Wasabi extract (6-MITC) was used as the research object. LPS induced BV2 cells as a neuroinflammatory cell model, and the anti-neuronal effect of wasabi extract was explored, which provided experimental basis and theoretical reference for its use in food prevention of neurological diseases.

\section{Materials and methods}

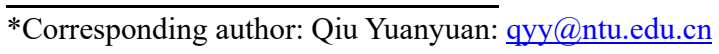




\subsection{Main materials}

Wasabi extract-6-MITC (Abcam); BV2 cells (basic bank of the Chinese Academy of Medical Sciences); lipopolysaccharide (L6529) (Sigma); MTT was purchased from Sigma; Taq SYBRGreenq PCRPremix was purchased from Roche;

\subsection{Cell culture and grouping}

BV2 cells were cultured in DMEM medium containing $10 \%$ fetal bovine serum at $37^{\circ} \mathrm{C}$ under $5 \% \mathrm{CO}_{2}$. The experiment was divided into three groups: (1) negative control group: cells without normal LPS treatment; (2) LPS treatment group (positive control group): $20 \mathrm{ng} / \mathrm{mL}$ LPS was added to the culture medium after 24 hours' normal culture, then damaged to have a treatment of 6 hours; (3) 6-MITC pre-protection group, after normal culture for $12 \mathrm{~h}, 0.2,1,2,4 \mu \mathrm{M} / \mathrm{mL}$ 6-MITC culture was added to the culture medium for $12 \mathrm{~h}$, and finally 20 $\mathrm{ng} / \mathrm{mL}$ LPS injury was added processing $6 \mathrm{~h}$.

\subsection{Cell survival rate detection}

When BV2 cells were implanted in 96-well plates and cells were $70 \%$ to $80 \%$ confluent, After the end of the cell treatment, add the final concentration at $0.5 \mathrm{mg} / \mathrm{mL}$ MTT solution and continue to culture it at $37^{\circ} \mathrm{C}, 5 \% \mathrm{CO} 2$ with 4 hours, and then add the $20 \%$ SDS solution for culturing another $20 \mathrm{~h}$ before measuring the absorbance at $570 \mathrm{~nm}$.

\subsection{Real-time fluorescence quantitative poly- merase chain reaction (RT-qPCR)}

The total RNA was extracted with Trizol reagent. The RNA concentration was determined by UV spectrophotometer before the reverse transcription into cDNA. The fluorescent PCR reaction was carried out by Bio-Rad fluorescence PCR instrument using SYBR green dye method. Primers were designed by using primer-Blast. Each set of experiments was repeated at least 3 times.

\section{Results}

\subsection{The effect of 6-MITC on BV2 cell morphology and cell viability}

The results are shown in Fig.2. The effect of 6-MITC $(0.2,1,4 \mu \mathrm{M} / \mathrm{mL})$ on the growth of BV2 cells. In the 6-MITC $0.2,1 \mu \mathrm{M} / \mathrm{mL}$ dose group, the microglia cell bodies were round and translucent, which was indistinguishable from the normal control group.

\subsection{The effect of LPS on cell viability}

In this paper, the activity of 6-MITC on LPS-damaged BV2 cells was detected by microplate reader. According to the pre-protected morphological analysis of the cells, we selected $0.2,1 \mu \mathrm{M} / \mathrm{mL}$ concentration for analysis, as shown in Fig.3, the cell viability of the model control group was significantly improved, and the cell differentia- tion after LPS treatment was pre-protected. The degree is not so large, so the activity tends to the normal group. We hypothesize that it is likely that the treatment of LPS stimulates BV2 cells to differentiate into M1 type, which makes cells more active.

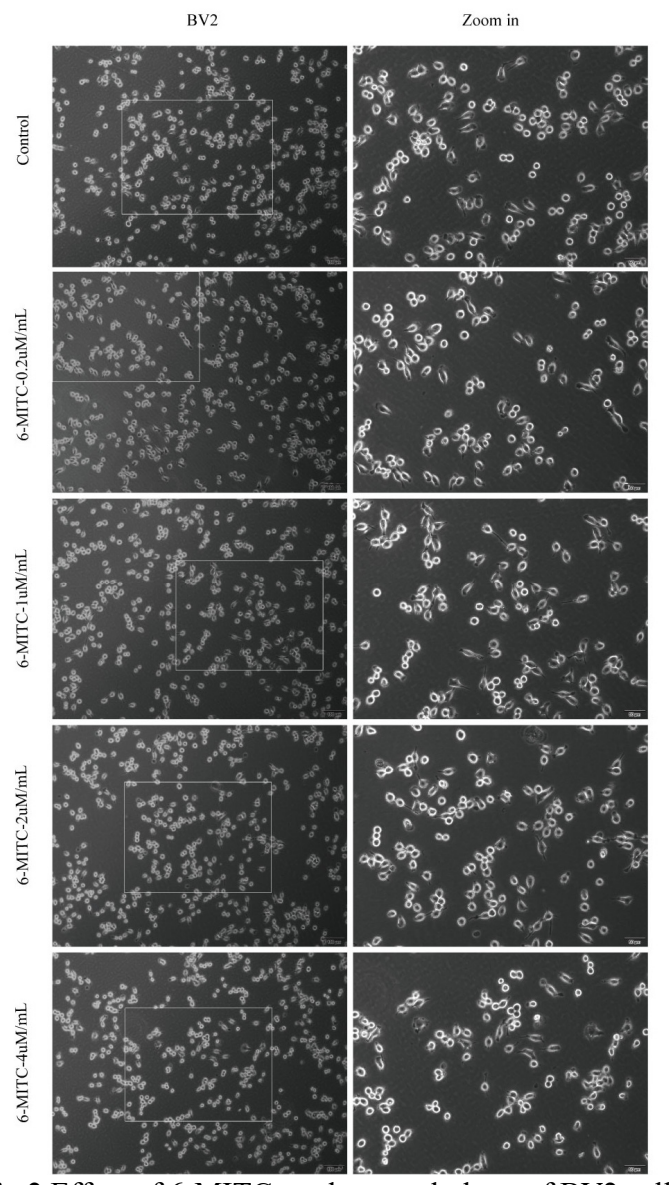

Fig.2 Effect of 6-MITC on the morphology of BV2 cells

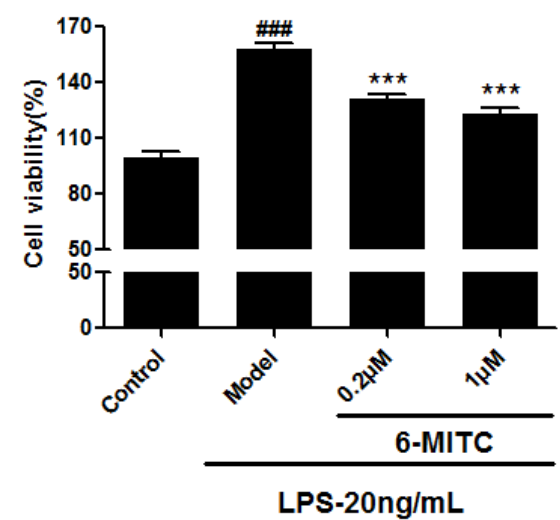

Fig.3 Effect of different concentrations of 6-MITC ( 0.2 $\mu \mathrm{M}, 1 \mu \mathrm{M})$ on the survival of LPS-treated BV2 cells. Mean $\pm \operatorname{SEM}(\mathrm{n}=10) . * * * \mathrm{P}<0.001$ vs model group; \#\#\# $\mathrm{P}<0.001$ vs control group.

\subsection{Effect of LPS on the morphology of BV2 microglia}

As shown in Fig.4, compared with the normal group, the cell bodies of the microglia treated only by LPS were 
significantly enlarged and darkened, and the branches increased and shown the typically activated microglia. The number of cells that were pre-protected for $12 \mathrm{~h}$ and then added the LPS lesion treatment was few activated .

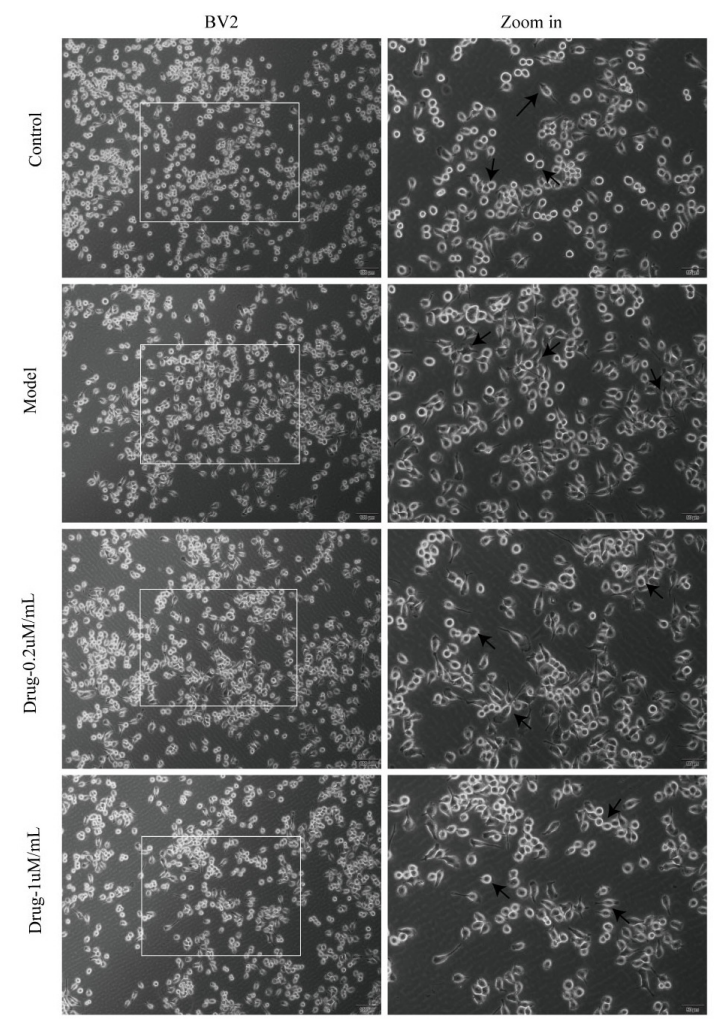

Fig.4 Changes of BV2 cells morphology after $6 \mathrm{~h}$ treatment with 6-MITC pretreatment for $12 \mathrm{~h}$

\subsection{RT-qPCR detection of 6-MITC on the expression of inflammatory cytokines mRNA in BV2 microglia under LPS}

The results are shown in Fig.4. When the concentration was $2,4 \mu \mathrm{M} / \mathrm{mL}$, the IL-6 mRNA expression level was excessively inhibited, so we used $0.2,1 \mu \mathrm{M} / \mathrm{mL}$ to treat the cells with to the results shown in Fig.5, The expression levels of cellular inflammatory factors IL-1 $\beta$, IL-6, TNF- $\alpha$ and iNOS were significantly up-regulated in the model group; After 6-MITC pretreatment, the mRNA expression of IL-1 $\beta$, IL- 6 , TNF- $\alpha$ and iNOS was down-regulated in the experimental group, and the concentration was statistically significant $(\mathrm{P}<0.05)$.

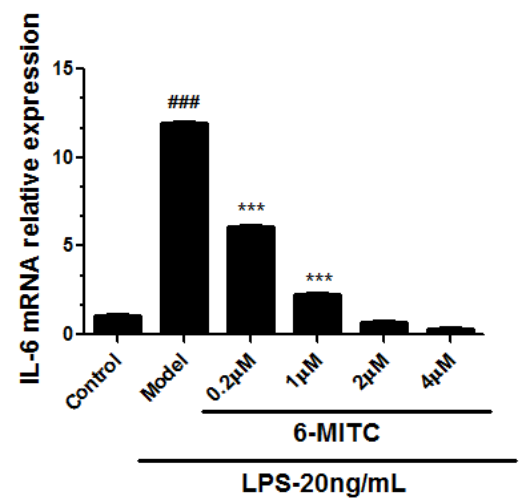

Fig.5 Effect of 6-MITC on IL-6 mRNA expression after LPS-induced BV2 cells activation. Mean \pm SEM (n $=3$ ). $* * * \mathrm{P}<0.001$ vs model group; \#\#\# $\mathrm{P}<0.001 \mathrm{vs}$

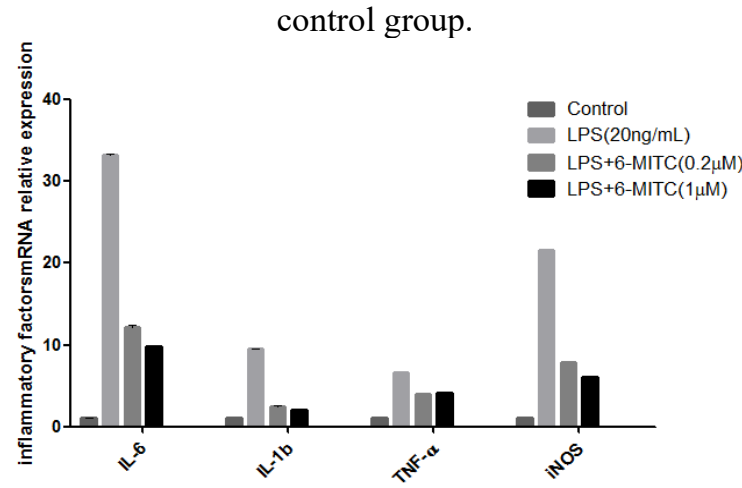

Fig.6 Effect of 6-MITC on LPS-induced inflammatory factor mRNA expression after activation of BV2 $\operatorname{cells}(n=3)$

\section{Discussion}

Recent studies have shown that 6-MITC has unique advantages in anti-inflammatory, anti-oxidant and anti-neurological diseases. Okamoto et $\mathrm{al}^{4}$ found that 6-MITC significantly inhibited leukocyte adhesion and decreased TNF- $\alpha$ induced IL-6 and monocyte chemoattractant protein-1 (MCP-1) expression in activated endothelial cells (EC). Takuhiro et $\mathrm{al}^{2}$ believed that 6-MITC blocked LPS-induced iNOS expression by blocking the Janus kinase 2 (Jak2) signaling cascade.It is accompanied by JNK-mediated transcription factor (Activator protein 1, AP-1) activation. Chen et $\mathrm{al}^{5}$ first revealed the gene expression profile of 6-MITC in inflammatory cell model macrophage RAW-264.7 by gene expression profiling in mouse macrophages by DNA microarray. 6-MITC can target immune and inflammation- related genes, including chemokines, interleukins and interferons to exert their anti-inflammatory functions. After LPS activates microglia, the mRNA expression levels of IL-1 $\beta$, IL-6, TNF- $\alpha$ and iNOS in cells are significantly increased, while the pretreatment with 6-MITC can significantly reduce the expression of inflammatory factors above. It is indicated that 6-MITC can inhibit the activation of microglia and reduce the production of inflammatory factors. Through this series of experiments to analyze the role of wasabi extract 6-MITC may inhibit the 
development of chronic neuroinflammation, it has provided not only a theoretical basis but also laid a solid foundation for the subsequent vivo experiments.

\section{References}

1. Trio PZ, Fujisaki S, Tanigawa S, et al.DNA Microar- ray Highlights Nrf2-Mediated Neuron Protection Targeted by Wasabi-Derived Isothiocyanates in IMR-32 Cells[J]. Gene Regul Syst Bio, 2016,10:73-83.

2. Uto T, Fujii M. Inhibition of lipopolysaccharide induced cyclooxygenase- 2 transcription by 6-(methyl- sulfinyl) hexyl isothiocyanate, a chemopreventive compound from Wasabia japonica (Miq.) Matsumura, in mouse macrophages[J]. Biochem Pharmacol 2005, 70(12): 1772-1784.

3. Boche D, Perry V H , Nicoll J A R . Activation patterns of microglia and their identification in the human brain[J]. Neuropathology \& Applied Neurobiology, 2013, 39(1):3-18.

4. Okamoto T, Akita N, Masashi Nagai. 6-Methylsulfinylhexyl isothiocyanate modulates endothelial cell function and suppresses leukocyte adhesion[J]. J Nat Med, 2014, 68(1):144-153.

5. Chen J, Uto T, Tanigawa S, et al. Microarray- based determination of anti-inflammatory genes targeted by 6-(methylsulfinyl)hexyl isothiocyanate in macrophages. [J]. Experimental \& Therapeutic Medicine, 2010, 1(1): 33-40. 